\title{
Clinical Relevance of Antiphospholipid Antibodies Levels During the Course of Severe COVID-19
}

\author{
Soumia Nachate ${ }^{1, *}$, Mahassine Moukaouim ${ }^{1}$, Loubna Darfaoui ${ }^{1}$, Zineb Nassiri $^{1}$, Imane Ibrahim $^{1}$, \\ Houssam Rebahi ${ }^{2}$, Hajar Chichou ${ }^{2}$, Abdelhamid Hachimi ${ }^{2}$, Mohamed-Abdenasser Semkaoui ${ }^{2}$, \\ Raja Hazime $^{1}$, Lamiae Essaadouni ${ }^{3}$, Brahim Admou ${ }^{1,4}$ \\ ${ }^{1}$ Laboratory of Immunology, Department of Biology, Mohamed VI University Hospital, Faculty of Medicine and Pharmacy of Marrakech, \\ Cadi Ayyad University, Marrakech, Morocco \\ ${ }^{2}$ Department of Anesthesia and Intensive Care, Mohamed VI University Hospital, Faculty of Medicine and Pharmacy of Marrakech, Cadi \\ Ayyad University, Marrakech, Morocco \\ ${ }^{3}$ Department of Internal Medicine, Mohamed VI University Hospital, Faculty of Medicine and Pharmacy of Marrakech, Cadi Ayyad \\ University, Marrakech, Morocco \\ ${ }^{4}$ B2S Research Laboratory, Mohamed VI University Hospital, Faculty of Medicine and Pharmacy of Marrakech, Cadi Ayyad University, \\ Marrakech, Morocco
}

\section{Email address: \\ docteurnachate@gmail.com(S. Nachate) \\ ${ }^{*}$ Corresponding author}

\section{To cite this article:}

Soumia Nachate, Mahassine Moukaouim, Loubna Darfaoui, Zineb Nassiri, Imane Ibrahim, Houssam Rebahi, Hajar Chichou, Abdelhamid Hachimi, Mohamed-Abdenasser Semkaoui, Raja Hazime, Lamiae Essaadouni, Brahim Admou. Clinical Relevance of Antiphospholipid Antibodies Levels During the Course of Severe COVID-19. International Journal of Immunology. Vol. 9, No. 2, 2021, pp. 37-40. doi: $10.11648 /$ j.iji.20210902.13

Received: April 18, 2021; Accepted: May 6, 2021; Published: May 14, 2021

\begin{abstract}
The aim of this study was to determine the clinical significance of antiphospholipid antibodies (APLs) during the follow-up of nine severe COVID-19 patients admitted to the Intensive Care Unit of the University Hospital. The measurement of APLs (IgG and IgM anti-cardiolipin (aCL) and anti- $\beta 2$-glycoprotein-1 (aB2GP1) was performed on the 1 st day and after 15 days of admission, using the chemiluminescence assay (threshold $=19 \mathrm{CU}$ ). The average age of patients was $64.7 \pm 20,44$ years (ranges: $30-88$ years), with a sex-ratio of 1.25. On day-1, APLs were positive in two cases, the first of which was positive for IgG aß2GP1 (94.9 CU) and IgG aCL (24.8 CU), and the second was positive only for IgG a $32 \mathrm{GP} 1$ (31.4 CU). On day-15, APLs showed negative results for both a $\beta 2 \mathrm{GP} 1$ and aCL for the first case, and decreasing titers of a $\beta 2 \mathrm{GP} 1$ for the second one. Interestingly, these two cases showed no thromboembolic events and had a good clinical outcome. Conversely, APL positivity occurred at day-15 in two cases, corresponding to IgG aB2GPI (49.3 CU) in one case, and IgG aCL (76 CU) in the other. Both cases presented with a prolonged activated-partial-thromboplastin-time, high levels of D-dimers and fibrinogen, associated with increased levels of ferritin and interleukin-6. Our series has shown that IgG aB2GPI or IgG aCL can be either transient or appear secondarily with significantly high titers. The latter condition was associated with a poor clinical outcome, which emphasizes the importance of APLs monitoring in severe COVID-19 as a potential prognostic factor.
\end{abstract}

Keywords: Severe COVID-19, Anti-phospholipid Antibodies, Monitoring, Prognosis

\section{Introduction}

Coronavirus disease 2019 (COVID-19) is associated with both severe systemic inflammation and a profound hypercoagulable state $[1,2]$. Abnormal coagulation parameters are associated with poor prognosis and high mortality in critically ill COVID-19 patients [3]. The association of a prolonged activated partial-thromboplastin time (aPTT) and thrombosis in some patients is a reminder of antiphospholipid syndrome (APS) wich is an autoimmune disorder characterized by thrombotic events occurring in patients with persistent APLs positivity [4, 5]. These 
auto-antibodies has been initially described in three patients presenting severe forms of COVID-19 associated with multiple cerebral infarctions raising the question of their role in the underlying mechanism of COVID-19-induced coagulopathy [6]. Since then, many reports have suggested a possible involvement of APLs in the pathophysiology and evolution of the disease [1]. The presence of APLs has been shown to be either associated with thrombotic complications or transient, resolving within a few weeks with no relevant clinical impact $[6,7,8]$. The aim of this study was to determine the clinical significance of APLs occurring during the follow-up of severe COVID-19 patients.

\section{Patients and Methods}

\subsection{Settings and Patient Population}

This study included severe COVID-19 patients admitted for up to 15 days in the Intensive Care Unit (ICU) for whom the SARS-COV-2 infection was confirmed by reverse-transcriptase-polymerase-chain-reaction (RT-PCR) on nasopharyngeal swabs. No patient had prior medical history of APS or other related auto-immune disease. All patients received thromboprophylaxis following current guidelines. Patients were assessed for APLs on day 1 and 15 days after ICU admission.

\subsection{Assessment of APLs Levels}

Based on IgG and IgM anti-cardiolipin (aCL) and anti- $\beta 2$-glycoprotein-1 (aB2GP1), APLs were measured using a chemiluminescence assay (BIO-FLASH ${ }^{\circledR}$, Inova Diagnostics, cut-off value $=20 \mathrm{CU})$. Were simultaneously recorded the patient's clinical data as well as biological parameters including: Cell blood count, Lactate dehydrogenase, Cardiac troponin I, aPTT, Fibrinogen, D-dimers, Ferritin, C-reactive protein and Interleukin-6.

\section{Results}

Nine patients have been enrolled whose mean age was 64.7 \pm 19.22 years (ranges: $30-88$ years), with a sex-ratio of 1.25 . The demographic characteristics, co-morbidities and clinical features of patients, as well as their laboratory results recorded on day 1 and day 15 are presented in table 1 and table 2 .

On day-1, APLs were positive in two cases (patient 1 and 2): the first case showed positivity for IgG aß2GP1 (94.9 CU) and $\operatorname{IgG}$ aCL (24.8 CU), and the second case was positive for only IgG a $32 \mathrm{GP} 1$ (31.4 CU). On day-15, among the nine monitored patients, APLs became negative for both a $32 \mathrm{GP} 1$ and $\mathrm{aCL}$ in the first case, and decreased in titer of a $32 \mathrm{GP} 1$ in the second case. These two cases had a good clinical outcome and didn't manifest thromboembolic events.

A positivity for IgG aB2GPI occurred on day-15 in one case (patient 3) and for IgG aCL in another case (patient 4), with titers of 49.3 and $76 \mathrm{CU}$ respectively. Both cases exhibited a prolonged aPTT, high levels of D-dimers and fibrinogen, associated with elevated levels of ferritin and interleukin-6, and finally died.

Table 1. Main Clinical and biological characteristics of 9 patients with severe COVID-19.

\begin{tabular}{|c|c|c|}
\hline \multicolumn{3}{|l|}{ Demographic characteristics } \\
\hline Mean age \pm SD (year) & $64,77 \pm 20,44$ & \\
\hline \multicolumn{3}{|l|}{ Gender (\%) } \\
\hline Male & 66,7 & \\
\hline Female & 33,3 & \\
\hline \multicolumn{3}{|l|}{ Medical history (\%) } \\
\hline Hypertension & 44,5 & \\
\hline Diabetes & 33,3 & \\
\hline Stroke & 11 & \\
\hline Coronary artery disease & 11 & \\
\hline Chronic obstructive pulmonary disease & 11 & \\
\hline Asthma & 11 & \\
\hline \multicolumn{3}{|l|}{ Symptoms (\%) } \\
\hline Fever & 77,8 & \\
\hline Cough & 88,9 & \\
\hline Dyspnea & 77,8 & \\
\hline Chest pain & 44,5 & \\
\hline Vomiting & 22,3 & \\
\hline Abdominal pain & 11 & \\
\hline \multicolumn{3}{|l|}{ Laboratory findings } \\
\hline & $1^{\text {st }}$ day & $15^{\text {th }}$ day \\
\hline White-cell count, median (IQR) (4000-10000 mm3) & $7590(2970-14570)$ & $7000(3620-99180)$ \\
\hline Lymphocytes, median (IQR) (1000-4000 mm3) & $800(520-2650)$ & $1920(1110-4220)$ \\
\hline Neutrophils, median (IQR) (2000-7500 mm3) & $5970(140-12070)$ & $4290(666-30540)$ \\
\hline Platelet count, median (IQR) (150000-450000 mm3) & $232(110-550)$ & $248(162-345)$ \\
\hline LDH, median (IQR) (0-250 U/liter) & $345(200-468)$ & $230(100-652)$ \\
\hline Cardiac troponin I, median (IQR) $(0-13 \mathrm{pg} / \mathrm{ml})$ & $11,8(4,02-26,17)$ & $116(3,54-116)$ \\
\hline
\end{tabular}




\begin{tabular}{lll}
\hline Demographic characteristics & & \\
\hline aPTT, median (IQR) (30-35 sec) & $27(20,6-32,10)$ & $22,5(21-43,60)$ \\
Fibrinogen, median (IQR) $(2-4 \mathrm{~g} /$ liter $)$ & $8(2,20-8)$ & $4(1,90-5,90)$ \\
D-dimer, median (IQR) $(0-0.5 \mathrm{mg} /$ liter $)$ & $3,64(0,57-28,47)$ & $1,65(0,78-21,27)$ \\
Ferritin, median (IQR) $(15-150 \mu \mathrm{g} /$ liter $)$ & $341(8,5-1170,40)$ & $409(13-3153)$ \\
C-reactive protein, median (IQR) $(0-5 \mathrm{mg} /$ liter $)$ & $121(4,64-282,88)$ & $195(1,51-195)$ \\
$\mathrm{IL}-6$, median $(\mathrm{IQR})(<7 \mathrm{pg} / \mathrm{ml})$ & $145(1,50-201)$ & $2592(5,58-5000)$ \\
\hline
\end{tabular}

* SD: standard deviation, IQR: interquartile range, LDH: Lactate dehydrogenase, aPTT: Activated partial thromboplastin time.

\section{Discussion}

After the emergence of the new coronavirus, thromboembolic complications were frequently reported especially in severe covid-19 patients and found in up to $58 \%$ of deceased ones [9], This has caused a great deal of concern among medical researchers to investigate the pathophysiological mechanism of this life-threatening condition. One of the hypotheses that have arisen is the involvement of APLs in this process. Until then, the debate is still controversial; there are competing assumptions from those who plead the participation of APLs in the genesis of coagulopathy associated with COVID-19 and those who defend that they are not implicated.

By monitoring APLs in severe COVID-19 patients, we observed two principal statuses: transient positivity of APLs with no clinical impact, and a secondary occurrence of $\mathrm{IgG}$ aß2GP1 and IgG aCL with high titers associated to a poor clinical course in two among nine monitored patients. This makes us suppose that APLs can be used as one of the markers to predict the prognosis in critically ill COVID-19 patients, whether or not they are involved in the occurrence of thromboembolic complications.

The first clinical condition characterized by the positivity of APLs since the initial admission (patients 1 and 2) may suggest pre-existing or concomitant APLs with the onset of COVID-19, which requires, on the one hand, an in-depth investigation of the medical history of the patients, and also suggests the potential role of SARS-CoV2 in inducing acute thromboembolic phenomena. A significant percentage of $57 \%$ and a large variety of APLs were detected in patients with severe COVID-19 [10]. These APLs may be associated with a hyperinflammatory state and thromboembolic events. The clinical and biological characteristics of the coagulopathy encountered in COVID-19 may resemble those of catastrophic antiphospholipid syndrome, but these two entities appear to be pathophysiologically different [10]. APLs in COVID-19 patients are mainly directed against $\beta 2$ GPI but display an epitope specificity different from antibodies in antiphospholipid syndrome [4]. Also, patients hospitalized in medicine wards for non-severe COVID-19 have been found to have APLs in $64 \%$ of cases, even with mild or transient levels, and these were significantly associated with the occurrence of thrombotic events [11].

Table 2. Antiphospholipid antibodies results at day-1 and day-15 of the monitoring.

\begin{tabular}{|c|c|c|c|c|c|c|c|c|c|c|c|c|c|c|c|c|c|c|}
\hline \multicolumn{19}{|l|}{ Antiphospholipid antibodies } \\
\hline & \multicolumn{2}{|c|}{ Patient 1} & \multicolumn{2}{|c|}{ Patient 2} & \multicolumn{2}{|c|}{ Patient 3} & \multicolumn{2}{|c|}{ Patient 4} & \multicolumn{2}{|c|}{ Patient 5} & \multicolumn{2}{|c|}{ Patient 6} & \multicolumn{2}{|c|}{ Patient 7} & \multicolumn{2}{|c|}{ Patient 8} & \multicolumn{2}{|c|}{ Patient 9} \\
\hline & $1^{\text {st }}$ & $15^{\text {th }}$ & $1^{\text {st }}$ & $15^{\text {th }}$ & $1^{\text {st }}$ & $15^{\text {th }}$ & $1^{\text {st }}$ & $15^{\text {th }}$ & $1^{\text {st }}$ & $15^{\text {th }}$ & $1^{\text {st }}$ & $15^{\text {th }}$ & $1^{\text {st }}$ & $15^{\text {th }}$ & $1^{\text {st }}$ & $15^{\text {th }}$ & $1^{\text {st }}$ & $15^{\text {th }}$ \\
\hline Anti & $-*$ & - & - & - & - & - & - & - & - & - & - & - & - & - & - & - & - & - \\
\hline Antica & 24.8 & - & - & - & - & - & - & 7( & - & - & - & - & - & - & - & - & - & - \\
\hline $\mathrm{M}(\mathrm{CU})$ & - & - & - & - & - & - & - & - & - & - & - & - & - & - & - & - & - & - \\
\hline anti- $\beta 2$-glycoprotein I IgG (CU) & 94.9 & - & 31.4 & 21.1 & - & 49.8 & - & - & - & - & - & - & - & - & - & - & & - \\
\hline Clinical course & \multicolumn{2}{|c|}{ Favorable } & \multicolumn{2}{|c|}{ Favorable } & \multicolumn{2}{|c|}{ Death } & \multicolumn{2}{|c|}{ Death } & \multicolumn{2}{|c|}{ Favorable } & \multicolumn{2}{|c|}{ Favorable } & \multicolumn{2}{|c|}{ Favorable } & \multicolumn{2}{|c|}{ Favorable } & \multicolumn{2}{|c|}{ Favorable } \\
\hline
\end{tabular}

* CU: Chemiluminescence Unit, (-): $<20 \mathrm{CU}$.

In a recently published cohort of 172 COVID-19 patients, a positivity of APLs was observed in many patients since the first day of hospitalization, with variable titers [12]. The authors did not find a pre-existing APL syndrome, but demonstrated that the APLs associated with SARS-CoV2 are potentially pathogenic. Using mouse models, purified IgG APLs fractions from COVID-19 patients induced neutrophil activation similarly to those isolated from individuals with established APS [12]. Moreover, neutrophil mediators have been shown to promote inflammation and microvascular thrombosis during COVID-19 [13].

Interestingly, our first two cases with positive APLs at day-1 had no thrombotic complications, but had biological abnormalities as well as mild APLs levels, which turned to negative at day- 15 in one case while the second case remained slightly positive but around the cut-off. Then, it is legitimate to raise the question whether the anticoagulant treatment that had been administered to our patients prevented the occurrence of thrombotic events. Similarly, Devreese et al, reported two cases of triple positive APLs (IgG aCL, IgG aB2GP1 and Lupus anti-coagulant) among 31 COVID-19 patients. When retested after one month, the first patient whose $\mathrm{IgG}$ aCL were mildly positive became negative and his IgG aB2GP1 persisted with lower titers. In the second patient, repeated testing was negative. Both patients had no thromboembolic complications and showed good clinical outcome [14]. APLs show a low prevalence in COVID-19 patients and are not associated with major thrombotic events $[4,15]$. Indeed, multiple acute infections, inflammation or thrombosis are known to trigger transient antiphospholipid antibodies $[15,16$, 
17], which must be taken into consideration to cautiously interpret the results of these auto-antibodies in general and establish their real clinical relevance [18].

In a Chinese study [7], Among three critical groups with similar clinical and laboratory features, the authors concluded that APLs may be helpful in predicting the poor clinical outcome if they are multiple and at least one APL is at moderate titers ( $>40$ Units). Moderate and high titers of APLs correlate with various clinical and biological parameters [13]. Furthermore, the level of IgG aCL autoantibodies has been reported as an independent risk factor for COVID-19 severity, and could serve as a simple strategy to stratify COVID-19 patients according to disease severity and help in the therapeutic decision process [19].

Similarly, our two deceased patients had either IgG aB2GP1 or IgG aCL with moderate $(49,8 \mathrm{CU})$ and high $(76$ $\mathrm{CU})$ titers respectively.

\section{Conclusions}

Our series revealed two main profiles of APL occurring in severe COVID-19: a favourable course in two cases of transient APL and a pejorative course for the two cases whose APL have risen significantly during the course of the disease. These findings consolidate the few data reported in literature. However, the clinical significance of these auto-antibodies remains poorly understood and requires more dedicated investigations on larger sample size with a long-term monitoring, including lupus anti-coagulant and even other APL specificities, in order to improve our knowledge about the potential pathogenic role of APL in COVID-19.

\section{Disclosure Statement}

The authors declare that they have no competing interests

\section{References}

[1] Valencia Manrique J, Ghosh K, Boma N. (2021) Anticardiolipin antibodies and COVID - 19 - A case report from America. J Med Virol. 93, 76-77.

[2] Sung J, Anjum S. (2020). Coronavirus Disease 2019 (COVID-19) Infection Associated With Antiphospholipid Antibodies and Four-Extremity Deep Vein thrombosis in a Previously Healthy Female. Cureus. 12 (6): e8408.

[3] Tang N, Li D, Wang X, Sun Z. (2020). Abnormal coagulation parameters are associated with poor prognosis in patients with novel coronavirus pneumonia. J Thromb Haemost. 18 (4): 844-847.

[4] Borghi MO, Beltagy A, Garrafa E, Curreli D, Cecchini G, Bodio C. (2020). Anti-Phospholipid Antibodies in COVID-19 Are Different From Those Detectable in the AntiPhospholipid Syndrome. Front. Immunol. 11: 584241.

[5] Tektonidou MG, Andreoli L, Limper M, et al. (2019). EULAR recommendations for the management of antiphospholipid syndrome in adults. Annals of the Rheumatic Diseases. 78 (10): 1296-1304.
[6] Zhang Y, Xiao M, Zhang S, Xia P, Cao W, Jiang W, et al. (2020). Coagulopathy and Antiphospholipid Antibodies in Patients with Covid-19. N Engl J Med. 382 (17): e38.

[7] Xiao M, Zhang Y, Zhang S, Qin X, Xia P, Cao W, et al. (2020) Brief Report: Anti-phospholipid antibodies in critically ill patients with Coronavirus Disease 2019 (COVID-19). Arthritis Rheumatol. 72(12): 1998-2004.

[8] Pineton de Chambrun M, Frere C, Miyara M, Amoura Z, Martin-Toutain I, Mathian A, et al. (2021). High frequency of antiphospholipid antibodies in critically ill COVID-19 patients: a link with hypercoagulability? (Letter To The Editor). Journal of Internal Medicine. 289: 422-424.

[9] Wichmann D, Sperhake J-P, Lütgehetmann M, Steurer S, Edler C, Heinemann A, et al. (2020). Autopsy findings and venous thromboembolism in patients with COVID-19. Ann Intern Med. 173 (4): 268-77.

[10] Amezcua-Guerra LM, Rojas-Velasco G, Brianza-Padilla M, et al. (2021). Presence of antiphospholipid antibodies in COVID-19: a case series study. Annals of the Rheumatic Diseases. 80: e73.

[11] Le Joncour A, Frere C, Martin-Toutain I. (2021). Antiphospholipid antibodies and thrombotic events in COVID-19 patients hospitalized in medicine ward. Autoimmun Rev. 20 (2): 102729.

[12] Zuo Y, Estes SK, Gandhi AA, Yalavarthi S, Ali RA, Lezak SP, et al. (2020). Prothrombotic autoantibodies in serum from patients hospitalized with COVID-19. Sci Transl Med. 12 (570): eabd3876.

[13] Zuo Y, Kanthi Y, Knight JS, Zuo Y, Yalavarthi S, Shi H, et al. (2020). Neutrophil extracellular traps in COVID-19 Neutrophil extracellular traps in COVID-19. JCI Insight. 5 (11): e138999.

[14] Devreese KMJ, Linskens EA, Benoit D, Peperstraete H. (2020). Antiphospholipid antibodies in patients with COVID-19: A relevant observation? J Thromb Haemost. 18 (9): 2191-2201.

[15] Galeano-Valle F, Oblitas CM, Ferreiro-Mazón MM, Alonso-Muñoz J, Del Toro-Cervera J, di Natale M, Demelo-Rodríguez P. (2020). Antiphospholipid antibodies are not elevated in patients with severe COVID-19 pneumonia and venous thromboembolism. Thromb Res. 192: 113-115.

[16] Mendoza- Pinto C, García- Carrasco M, Cervera R. (2018). Role of infectious diseases in the antiphospholipid syndrome (including its catastrophic variant). Curr Rheumatol Rep. 20, 62.

[17] Abdel-Wahab N, Talathi S, Lopez-Olivo MA, Suarez-Almazor ME. (2018). Risk of developing antiphospholipid antibodies following viral infection: a systematic review and meta-analysis. Lupus. 27, 572-583.

[18] Mehta S, Bhandari S, Mehta S. (2020). Cautious interpretation of antiphospholipid antibodies in COVID-19. Clinica Chimica Acta. 509, 166.

[19] Bertin D, Brodovitch A, Beziane A, Hug S, Bouamri A, Mege J. L, et al. (2020), Anticardiolipin IgG Autoantibody Level Is an Independent Risk Factor for COVID - 19 Severity. Arthritis Rheumatol. 72: 1953-1955. 